Check for updates

Cite this: RSC Adv., 2018, 8, 36534

Received 3rd September 2018 Accepted 21st October 2018

DOI: $10.1039 / \mathrm{c} 8 \mathrm{ra07342b}$

rsc.li/rsc-advances

\section{A targeted metabolomic protocol for quantitative analysis of volatile organic compounds in urine of children with celiac disease}

\begin{abstract}
Natalia Drabińska, (DD *a Hafiz Abdul Azeem (D) ${ }^{b}$ and Urszula Krupa-Kozak (D) ${ }^{a}$
Volatile organic compounds (VOCs) in biological samples have gained popularity for disease monitoring and diagnosis. Celiac disease (CD) is one of the many prevalent health conditions which are challenging to diagnose. The aim of this study was to optimize a solid phase microextraction followed by gas chromatography-mass spectrometry, for quantitative analysis of a wide range of VOCs in the urine of patients with $C D$. Multivariate design of experiment was used to optimize the extraction conditions for the analysis of 15 urinary VOCs. Based on the performed experiments, extraction using $2.98 \mathrm{~g}$ of sodium chloride and $21 \mu \mathrm{L}$ of $6 \mathrm{M}$ hydrochloric acid for $15 \mathrm{~min}$ at $30^{\circ} \mathrm{C}$, using a CAR/PDMS fiber in headspace mode was found to be the most effective procedure for the analysis of the selected biomarkers. It was also demonstrated that the proposed method could distinguish between children with CD and healthy children based on the profile of VOCs. It is believed that quantitative analysis of these biomarkers will extend our understanding of $C D$ and could be used for monitoring in patients under treatment.
\end{abstract}

\section{Introduction}

Celiac disease (CD) is an autoimmune, gluten-induced enteropathy, observed in genetically predisposed individuals, and affects approximately $1 \%$ of the global population. ${ }^{1} \mathrm{CD}$ often remains undiagnosed due to its atypical and silent manifestations. ${ }^{2}$ The only available treatment of $\mathrm{CD}$ is to follow a glutenfree diet, however, many patients continue to experience symptoms of the disease even after years of an appropriate dietary compliance. It is believed that more knowledge is required for better understanding, screening and monitoring of $\mathrm{CD}$, consequently improving the gluten-free diet, alleviating the symptoms of the disease.

Analysis of volatile organic compounds (VOCs) in urine samples holds promise for non-invasive detection of the medical conditions. ${ }^{3}$ VOCs are a diverse group of carbon-based compounds that are released by the human body as by-products of metabolism, hormonal changes, microbiota activity and presence of environmental pollutants. ${ }^{4}$ They can be detected in all biological specimens including breath, blood, saliva, urine, feces and sweat. ${ }^{5}$ There is an increasing number of studies analysing urine for detection of various health conditions due to the ease of sampling without compromising the comfort of

\footnotetext{
${ }^{a}$ Department of Chemistry and Biodynamics of Food, Institute of Animal Reproduction and Food Research of Polish Academy of Sciences, Tuwima 10 Str., 10-748 Olsztyn, Poland.E-mail: n.drabinska@pan.olsztyn.pl; natalia_drabinska@wp.pl; Fax: +48 89 52401 24; Tel: +48 895234639

${ }^{b}$ Centre for Analysis and Synthesis, Department of Chemistry, Lund University, Naturvetarvägen 14/Sölvegatan 39 A, 22100 Lund, Sweden
}

patients even with repeated sampling. ${ }^{6}$ Analysis of VOCs in urine can provide information about changes in metabolism caused by the presence of the disease. Urinary biomarkers, including VOCs, were reported for the detection of cancer, ${ }^{7,8}$ oxidative stress, ${ }^{9}$ inflammatory bowel disease,${ }^{10}$ urological ${ }^{11}$ and infectious diseases. ${ }^{12}$ Fewer studies have reported changes in the profile of VOCs in urine and fecal samples in patients with $\mathrm{CD}$, compared to healthy individuals. ${ }^{13,14}$ Moreover, the analysis of VOC in urine has been used to distinguish people with CD from those with irritable bowel syndrome. ${ }^{15}$ Although, interest in the analysis of urinary VOCs is growing, scarce information is available on the quantitative analyses of individual compounds or selected classes of metabolites of urinary biomarkers, ${ }^{6,9,16,17}$ highlighted by Mochalski and Unterkofler. ${ }^{6}$ Since VOCs serve as markers of bodily biological mechanisms, a change in their relative concentrations may provide a deeper understanding of different stages and propagation of a disease. Therefore, it is imperative to develop simple and robust analytical methods for the quantitative analysis of a wide range of biomarkers from urine for patients with CD.

A number of analytical methods have been used to study the composition of urine, including field asymmetric ion mobility spectrometry, ${ }^{15}$ sensor arrays (e.g. e-nose) ${ }^{18}$ nuclear magnetic resolution spectroscopy, ${ }^{19}$ ion flow tube mass spectrometry ${ }^{20}$ and most commonly gas chromatography-mass spectrometry (GCMS) with various extraction techniques. ${ }^{6,8,9,21}$ Urine contains a wide range of metabolites including ketones, alcohols, pyrroles and sulfur-containing compounds. ${ }^{5}$ The diversity of VOCs in terms of chemical properties, functional groups and polarities presents challenges that need to be addressed by 
Table 1 Metabolites investigated in the present study with their CAS number, retention time (RT) and calculated retention index (RI)

\begin{tabular}{llll}
\hline Compound & CAS number & RT (min) & RI \\
\hline 2,3-Butanedione & $431-03-8$ & 11.45 & 646 \\
2-Butanone & $78-93-3$ & 11.74 & 653 \\
Thiophene & $110-02-1$ & 14.09 & 707 \\
Dimethyl disulfide & $624-92-0$ & 17.44 & 790 \\
Hexanal & $66-25-1$ & 19.80 & 850 \\
4-Methylthiazole & $693-95-8$ & 20.59 & 869 \\
4-Heptanone & $123-19-3$ & 22.61 & 921 \\
2-Heptanone & $110-43-0$ & 23.48 & 945 \\
2-Pentylfuran & $3777-69-3$ & 26.16 & 1017 \\
Dimethyl trisulfide & $3658-80-8$ & 26.88 & 1038 \\
6-Methyl-5-hepten-2-one & $110-93-0$ & 27.11 & 1045 \\
Benzaldehyde & $100-52-7$ & 27.24 & 1048 \\
Octanal & $124-13-0$ & 27.52 & 1057 \\
trans-3-Octen-2-one & $18402-82-9$ & 29.29 & 1107 \\
$p$-Cresol & $106-44-5$ & 32.46 & 1206 \\
1,3-Di-tert-butylbenzene & $1014-60-4$ & 34.99 & 1290 \\
& & & \\
\hline
\end{tabular}

more sensitive analytical methods. In the present study, solid phase microextraction (SPME) was optimized and validated followed by GCMS, for quantitative analysis of a wide range of urinary biomarkers in patients with CD. SPME is a robust sample treatment technique which offers flexibility to be finetuned for quantitative analysis of a wide range of organic compounds $\mathrm{s}^{22}$ at trace levels. The extraction method was optimized by multivariate design of experiment (DoE) for 15 urinary biomarkers representing various classes of organic compounds. The biomarkers were chosen on the basis of previously published research on the most dominant differences between the metabolic profiles of patients with $\mathrm{CD}$ and healthy individuals. ${ }^{13}$ The VOCs studied here comprised of 2,3-butanedione; 2butanone; thiophene; dimethyl disulfide; hexanal; 4-heptanone; 2-heptanone; 2-pentylfuran; dimethyl trisulfide; 6-methyl5-hepten-2-one; benzaldehyde; octanal; trans-3-octen-2-one; $p$ cresol and 1,3-di-tert-butylbenzene (Table 1). It is believed that quantitative analysis of these biomarkers will extend our understanding not only towards the propagation of $\mathrm{CD}$ but also be useful to monitor the condition of patients under treatment. Moreover, in this study, the use of the optimized method for the analysis of real samples from healthy and CD children was demonstrated. To our knowledge, this is the first ever study dedicated to the quantitative analysis of urinary volatile biomarkers for CD.

Hypothesis: children with $\mathrm{CD}$ can be distinguished from healthy children based on the profile of urinary VOCs.

\section{Experimental}

\section{A. Standards and materials}

Standards of all analyzed compounds: 2,3-butanedione, 2butanone, thiophene, dimethyl disulfide, hexanal, 4-heptanone, 2-heptanone, 2-pentylfuran, dimethyl trisulfide, 6methyl-5-hepten-2-one, benzaldehyde, octanal, trans-3-octen2-one, $p$-cresol and 1,3-di-tert-butylbenzene, internal standard: 4-methyl-2-phenol and saturated alkanes $\left(\mathrm{C}_{7}-\mathrm{C}_{40}\right)$ were supplied by Sigma-Aldrich (Saint Louis, USA). Retention indexes for selected compounds were calculated according to Zellner and co-authors. ${ }^{23}$ MilliQ water (Millipore, Bedford, USA) was used for the preparation of standards. Sodium chloride ( $\mathrm{NaCl}, \geq 99.5 \%$ ) was purchased from Sigma-Aldrich (Saint Louis, USA), sodium hydroxide ( $\mathrm{NaOH}, 99 \%)$ from POCH (Gliwice, Poland) and hydrochloric acid ( $\mathrm{HCl}, 37 \%)$ was purchased from Chempur (Piekary Śląskie, Poland). The SPME fibers used in the experiments were: 50/30 $\mu \mathrm{m}$ divinylbenzene/ carboxen/polydimethylsiloxane (DVB/CAR/PDMS) (stable flex), $65 \mu \mathrm{m}$ polydimethylsiloxane/divinylbenzene (PDMS/DVB) (stable flex), $7 \mu \mathrm{m}$ polydimethylsiloxane (PDMS7), $100 \mu \mathrm{m}$ polydimethylsiloxane (PDMS100), $75 \mu \mathrm{m}$ carboxen/ polydimethylsiloxane (CAR/PDMS) (stable flex), $85 \mu \mathrm{m}$ polyacrylate (PA) (stable flex) and $60 \mu \mathrm{m}$ carbowax-polyethylene glycol (PEG), purchased from Supelco (Bellefonte, PA, USA).

\section{B. Sampling}

Urine samples were collected in the morning (before breakfast) from healthy children (5-13 years old; $50 \%$ girls) as well as children with CD (4-14 years old; $78 \%$ girls), 9 samples from each group. Approximately $30 \mathrm{~mL}$ of morning urine (second spontaneous urine) was collected. The samples were immediately divided into aliquots of $4 \mathrm{~mL}$ and stored at $-80{ }^{\circ} \mathrm{C}$ until further analysis. All procedures involving human participants were in accordance with the Bioethics Committee of the Faculty of Medical Sciences of the University of Warmia and Mazury in Olsztyn (agreement no. 23/2015 of 16 June 2015) and with the 1964 Helsinki declaration and its later amendments or comparable ethical standards. Informed consent was obtained from all individual participants included in the study.

\section{Preparation of calibration solutions and SPME fibers}

Standard stock solutions were prepared by dissolving $1 \mu \mathrm{L}$ of each reference standard ( $3 \mu \mathrm{L}$ for 2,3-butanedione; 2-butanone) in MilliQ water in a $50 \mathrm{~mL}$ measuring flask. The standard working solutions were obtained by diluting the stock solutions. 4-Methyl-2-pentanol was used as an internal standard (196.24 $\mathrm{nmol} \mathrm{L}{ }^{-1}$ ). All fibers were conditioned prior to analysis as per the manufacturer's recommendations.

\section{SPME optimization}

Qualitative variables such as the type of SPME fiber and mode of extraction including headspace (HS) and direct immersion (DI) were investigated. Seven fibers comprising PA, PDMS100, PDMS7, CAR/PDMS, PDMS/DVB, DVB/CAR/PDMS and PEG were tested for the selected biomarkers. Prior to the extractions, samples were incubated for $20 \mathrm{~min}$ at $50{ }^{\circ} \mathrm{C}$ with a shaking speed of $500 \mathrm{rpm}$ using MultiTherm shaker (Benchmark Scientific, Edison, USA). The extractions were then performed manually by inserting the fiber into the sample vials and exposing it for $20 \mathrm{~min}$. HS and DI experiments were performed using $4 \mathrm{~mL}$ and $9 \mathrm{~mL}$ of sample respectively, in $20 \mathrm{~mL}$ vials sealed with metal caps. $4 \mathrm{~mL}$ of sample was used in HS mode as reported by Mills et al. ${ }^{24} \mathrm{In}$ DI mode, $9 \mathrm{~mL}$ of sample was used to overcome practical limitations of submerging fiber into smaller 


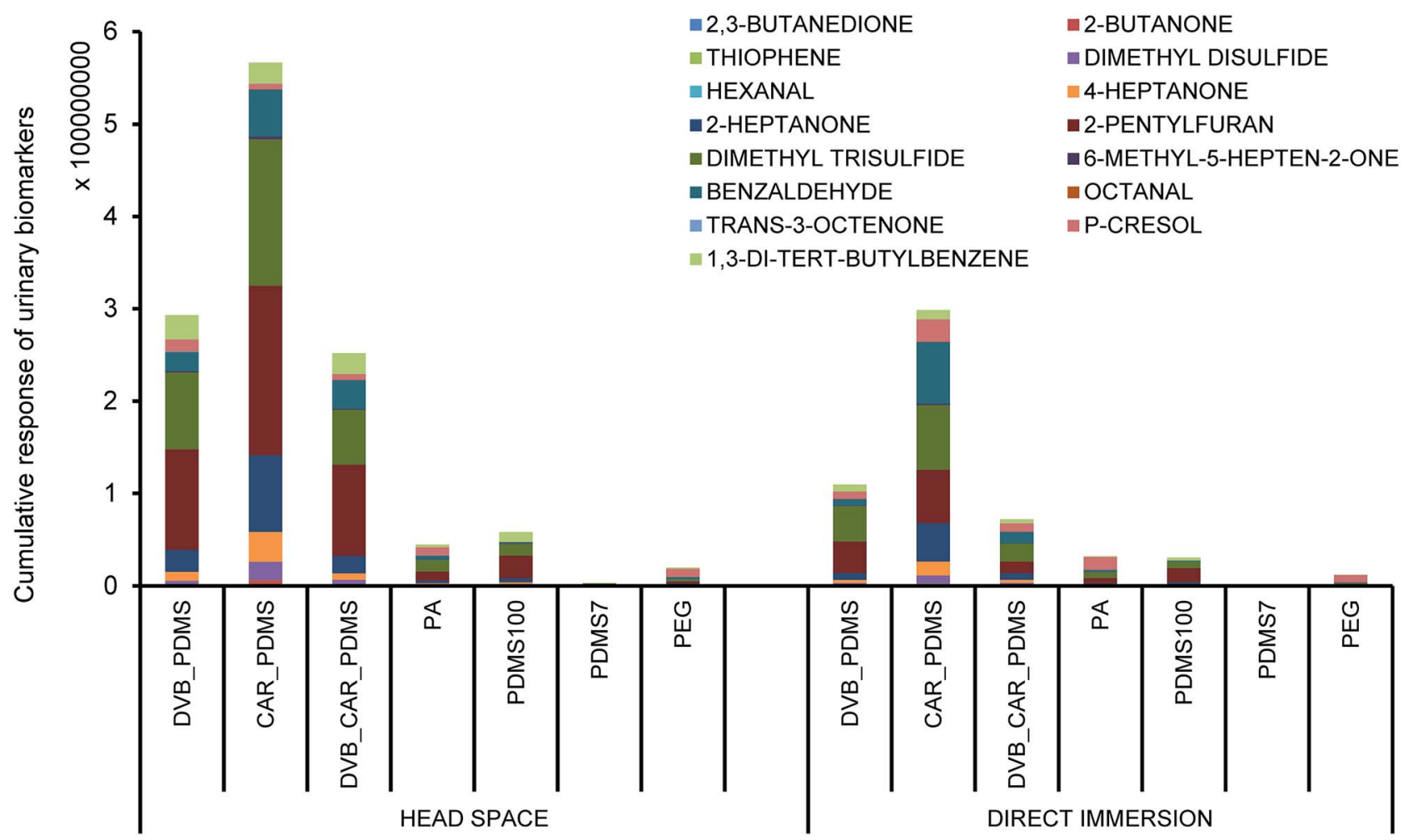

Fig. 1 Comparison of extraction performance of different SPME fibers for selected urinary biomarkers.

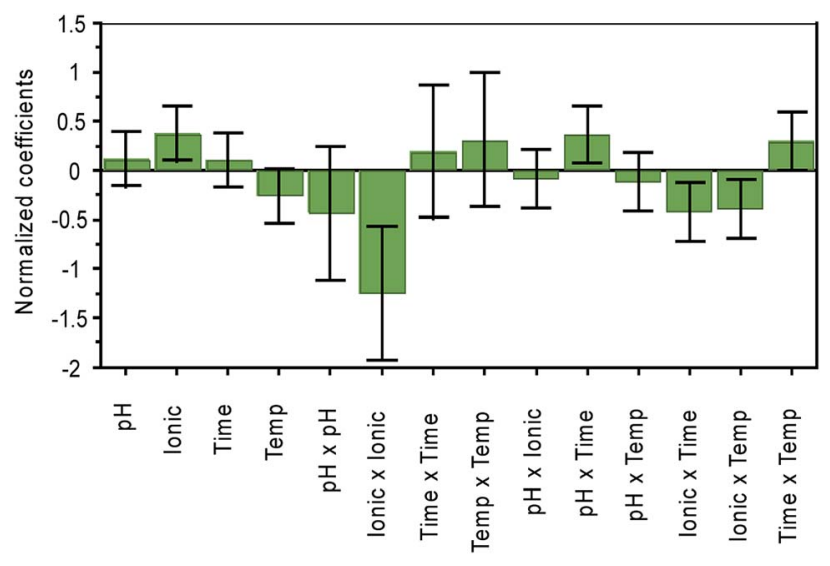

Fig. 2 Normalized influence of variables on the extraction of the urinary biomarkers. Ionic $=$ ionic strength; temp $=$ temperature.

samples. The values were normalized for comparison. After extractions, the fibers were introduced into the inlet of a gas chromatograph and the biomarkers were thermally desorbed for $10 \mathrm{~min}$ at $240{ }^{\circ} \mathrm{C}$ in a splitless mode..$^{25}$ The time of desorption was selected based on preliminary results obtained to avoid carryover. Screening experiments were performed in duplicates. The most suitable fiber coating and extraction mode were selected to optimize peak areas of individual compounds. Factors such as the amount of sample and headspace volume ( $4 \mathrm{~mL}$ of sample in $20 \mathrm{~mL}$ vial) were kept constant as described elsewhere. ${ }^{25,26}$
A DoE using MODDE 10.1.1 software (Umetrics, Umeå, Sweden) was employed to study interactions between different quantitative variable i.e. $\mathrm{pH}$, ionic strength, extraction time and temperature. A quadratic central composite faced (CCF) design including 27 experiments with 3 center points was used to optimize the variables. The lower and upper ranges of $\mathrm{pH}$ were maintained by the addition of $100 \mu \mathrm{L}$ of $6 \mathrm{M}$ hydrochloric acid and $100 \mu \mathrm{L}$ of $6 \mathrm{M}$ sodium hydroxide into $4 \mathrm{~mL}$ of the sample, respectively. The experimental ranges of ionic strength were between $0-4 \mathrm{~g}$ of sodium chloride added to the sample, extraction time between 15-60 min and temperature between $30-60{ }^{\circ} \mathrm{C}$. In CCF, as a standard procedure, the variables were optimized using the lower limits, the middle points and the upper limits of the experimental ranges.

\section{E. Gas chromatography-mass spectrometry}

Urinary biomarkers were analyzed using a HP 5972 mass selective detector coupled with a HP 5890 gas chromatograph (Agilent Technologies, Santa Clara, USA). The compounds were separated using a Zebron ZB-624 capillary column, $60 \mathrm{~m} \times$ $0.25 \mathrm{~mm} \times 1.40 \mu \mathrm{m}$, (Phenomenex, Torrance, United States of America). A $0.75 \mathrm{~mm}$ ID splitless glass liner (Supelco, Bellefonte, PA) was used with an inlet temperature of $240{ }^{\circ} \mathrm{C}$. The oven temperature program was $40{ }^{\circ} \mathrm{C}$ for 2 min then it was increased to $220{ }^{\circ} \mathrm{C}$ at a rate of $5{ }^{\circ} \mathrm{C}$ min and maintained for 5 min with a total run time of $42 \mathrm{~min}^{.5}$ The carrier gas was helium at a constant flow rate of $1 \mathrm{~mL} \mathrm{~min}^{-1}$. A solvent delay was set for 5 min. Mass spectra were obtained by electron ionization (EI) in the range of $40-550 \mathrm{~m} / \mathrm{z}$. Transfer line 
Table 2 Validation parameters calculated from a mixture of commercial standards. Compounds are ordered with respect to their increasing retention times

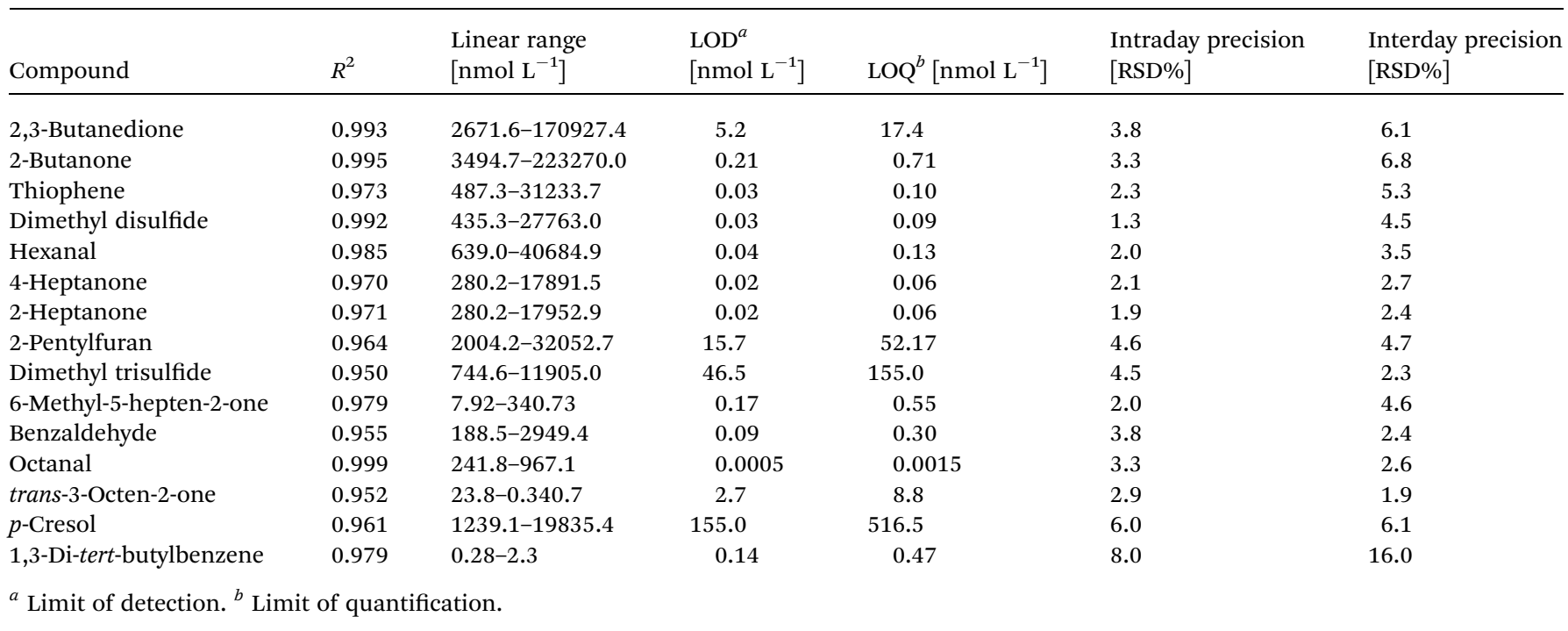

temperature, ion source temperature and quadrupole temperature were $240{ }^{\circ} \mathrm{C}, 230{ }^{\circ} \mathrm{C}$, and $180{ }^{\circ} \mathrm{C}$ respectively. Total ion chromatograms were analyzed with the MSD ChemStation E.02.02.1431 software (Agilent Technologies, Santa Clara, USA). Identification of compounds was performed by comparison of the retention times and $m / z$. Quantification of compounds was done by external standard calibration.

\section{F. Statistical analyses}

Real sample analysis was performed in duplicates. The normality of the quantitative variables was tested by the Shapiro-Wilk test. As the data showed non-normal distribution, quantitative variables are expressed as a median value (P25P75). Differences in the level of individual VOCs between healthy children and children with CD were tested with the nonparametric Mann-Whitney $U$ test. Results were considered statistically significant at the $5 \%$ critical level $(p<0.05)$. The analyses were performed using the Statistica 10.0 software (StatSoft, Tulsa, USA).

Partial least squares-discriminant analysis (PLS-DA) was performed to evaluate separation between healthy and CD children, considering the urinary VOC data. The analysis was performed using the XLSTAT statistical software for Microsoft Excel.

\section{Results and discussion}

\section{A. Screening experiments}

Screening experiments showed that the CAR/PDMS fiber was the most suitable in both HS and DI extraction mode. The fiber yielded the highest response for all candidate biomarkers than other fiber types (Fig. 1). The findings confirm the ability for CAR/PDMS fibers in extracting VOCs such as alcohols, sulfurcontaining compounds, carbonyl group-containing organic compounds and other volatile compounds from challenging matrices such as urine. There are reports using CAR/PDMS fiber for the analysis of VOCs with small molecular masses ${ }^{27}$ in urine samples for the profiling of potential urinary cancer biomarkers, ${ }^{7}$ VOCs in the urine of elderly men $^{28}$ and the human metabolome. ${ }^{19}$

A higher response to selected analytes was obtained by HS mode compared to DI for the majority of the fibers (Fig. 1). HS mode is commonly used for complex liquid and solid matrixes to overcome complications caused by dirty samples and to reduce sampling time. ${ }^{29}$ On the other hand, DI mode is preferred for the extraction of less volatile and more polar compounds. $^{22}$ Another disadvantage of DI mode is that a significantly higher sample volume is required for complete immersion of the fiber in the sample. Hence, CAR/PDMS fiber in HS mode was selected for further optimization of the extraction parameters.

\section{B. Method optimization}

Central Composite Faced (CCF) design was used to optimize four quantitative variables i.e. extraction time, temperature, ionic strength and $\mathrm{pH}$. The range of factors was selected based on the literature. ${ }^{26,30}$ A total explained variance of $90 \%\left[R^{2}(Y)=\right.$ $0.9]$ and a cross-validated predictability of $48 \%\left[Q^{2}(Y)=0.48\right]$ was observed in the optimized CCF model. The normalized influence of variables including extraction time, temperature, amount of salt and $\mathrm{pH}$ is shown in Fig. 2.

The ionic strength was found to be the most influential variable on extraction efficiency. Depending on the nature of the compound, the addition of salt can influence the extraction efficiency both positively and negatively as presented by Risticevic et $a l .{ }^{22}$ The addition of salt facilitates the transfer of the molecules from the sample into the HS and ultimately to the fiber surface, increasing the overall sensitivity. However, too high ionic strength may result in electrostatic interactions of 
a)

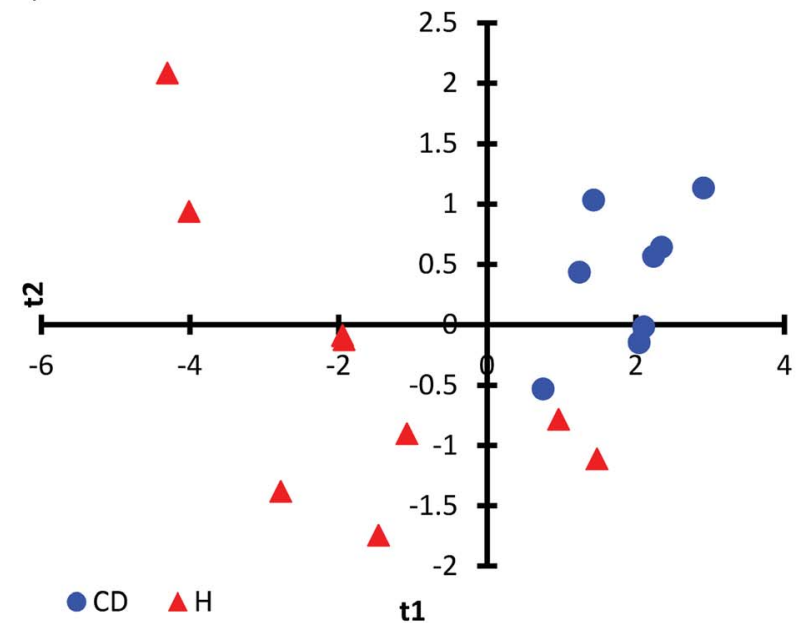

b)

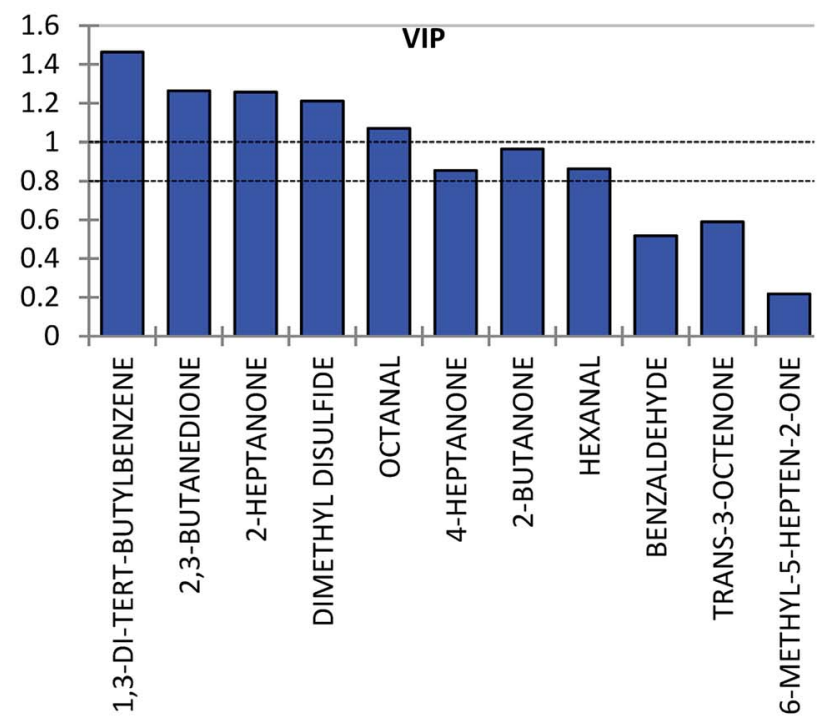

Variable

Fig. 3 (a) PLS-DA scores plot (LV2, $R^{2} X=0.491 ; R^{2} Y=0.609 ; Q^{2}=$ $0.395)$ obtained for target analysis between healthy (red triangles) and CD children (blue circles). (b) Variable importance in projection (VIP) plots for partial least squares analysis. A VIP value larger than 0.83 indicates the contribution of VOC in separating the groups (above the line).

the compound with the salt molecules, reducing the ability of the metabolite to pass into the HS. ${ }^{27}$ In the present study, the type of salt was not investigated, and sodium chloride was used since it is the most commonly used salt in earlier studies on urine..$^{7,25,26,30}$ In our study, $2.98 \mathrm{~g}$ of salt per $4 \mathrm{~mL}$ of urine sample was found to provide the optimum ionic strength.

It was found that $\mathrm{pH}$ had no significant effect on extraction efficiency (Fig. 2). The compounds analyzed by SPME can only adsorb onto the fiber surface from HS if they are present in a neutral form; in the selected matrix not all compounds are present in the neutral form. To achieve the conversion of molecules into the neutral form, the $\mathrm{pH}$ was adjusted. ${ }^{22}$ The extraction of acidic compounds is improved at a lower $\mathrm{pH}$, whilst a high $\mathrm{pH}$ improves the extractability of basic compounds. Based on the results obtained by CCF, the $\mathrm{pH}$ was adjusted by adding $21 \mu \mathrm{L}$ of $6 \mathrm{M}$ hydrochloric acid.

Surprisingly, extraction time and temperature were found to be less significant factors than ionic strength, contrary to what was expected from the literature. ${ }^{16}$ Fig. 2 shows a normalized coefficient plot with the relative influence of different variables on extraction efficiency. A temperature of $30{ }^{\circ} \mathrm{C}$ was found to be the most suitable for the selected VOCs. The most suitable extraction time was 15 minutes, which could be explained by the volatile nature of the biomarkers.

Finally, the optimal conditions for the extraction and quantification of selected urinary biomarkers of $\mathrm{CD}$ were a temperature of $30{ }^{\circ} \mathrm{C}$, and an extraction time of $15 \mathrm{~min}$ with the addition of $2.98 \mathrm{~g}$ of sodium chloride and $21 \mu \mathrm{L}$ of $6 \mathrm{M}$ hydrochloric acid in $4 \mathrm{~mL}$ of sample.

\section{Analytical performance}

The method was evaluated for inter and intraday precision, limits of detection (LOD), limits of quantification (LOQ) and linearity of the method for all 15 biomarkers. Furthermore, all the analyses were performed in triplicate and the peak areas of standards were normalized relative to the peak area of the internal standard. The parameters of method performance for each selected VOC using the developed method are presented in Table 2.

Method linearity was determined by evaluating the regression curves of standard solutions and expressed by the squared determination coefficient $R^{2}$. The method was found to have a linear response $\left(R^{2}\right.$ in a range of $\left.0.950-0.999\right)$ for all the selected VOCs, their ranges are given in Table 2 .

Interday precision was calculated by analyzing a batch of six standard mixtures. Intraday precision was determined by analyzing another batch of six standard mixtures the following day. Precision was expressed as relative standard deviation (RSD). In general, except for 1,3-di-tert-butylbenzene, the intraday precision was below $5 \%$ for the rest of the analytes, while interday precision ranged from 1.9 to $6.8 \%$ RSD, which was found as satisfactory.

LOD and LOQ were calculated by analyzing the signal-tonoise ratio $(\mathrm{S} / \mathrm{N})$. The $\mathrm{LOD}$ values were found to be $<0.3 \mathrm{nmol}$ $\mathrm{L}^{-1}$ for all the biomarkers except 2,3-butanedione, 2-pentylfuran, dimethyl trisulfide, trans-3-octen-2-one and $p$-cresol. Upon comparison with available methods, the optimized SPME method was found to be more sensitive for some of the candidate biomarkers analyzed by already available methods. The LOD for hexanal analyzed by HS combined with GCMS was 0.60 nmol L ${ }^{-1}$ (ref. 9) (in our study $0.04 \mathrm{nmol} \mathrm{L}^{-1}$ ). In another study using SPME with an additional derivatization step, the LOD for

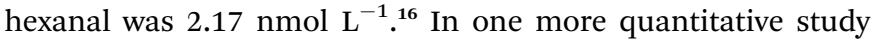
optimizing SPME with focus on hexanal and heptanal, the LOD

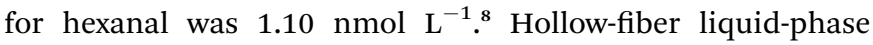
microextraction followed by capillary electrophoresis, ${ }^{\mathbf{3 1}}$ and magnetic solid-phase extraction followed by high performance liquid chromatography ${ }^{32}$ were used for quantitative analysis of hexanal and heptanal, and the obtained LOD was $2.7 \mathrm{nmol} \mathrm{L}^{-1}$ 
Table 3 Concentration ( $\mathrm{nmol} \mathrm{L}{ }^{-1}$ ) of selected VOCs in urine of healthy and CD children to distinguish between the two groups

\begin{tabular}{|c|c|c|c|c|c|c|c|}
\hline \multirow[b]{2}{*}{ Compound } & \multicolumn{3}{|c|}{ Healthy children } & \multicolumn{3}{|c|}{ Celiac disease children } & \multirow[b]{2}{*}{$p$-Value } \\
\hline & Median & $\mathrm{P} 25$ & P75 & Median & $\mathrm{P} 25$ & P75 & \\
\hline 2-Butanone & 5539.2 & 3449.2 & 11270.7 & 801.7 & 663.8 & 2079.1 & 0.0141 \\
\hline Thiophene & $\mathrm{ND}^{a}$ & & & ND & & & \\
\hline Dimethyl disulfide & 386.5 & 232.1 & 530.7 & 96.0 & 73.3 & 154.0 & 0.0108 \\
\hline 2-Heptanone & 67.5 & 39.5 & 99.0 & 7.9 & 4.4 & 9.5 & 0.0108 \\
\hline 2-Pentylfuran & $\mathrm{NQ}^{b}$ & & & NQ & & & \\
\hline Dimethyl trisulfide & NQ & & & NQ & & & \\
\hline 6-Methyl-5-hepten-2-one & 9.1 & 7.3 & 16.2 & 15.4 & 15.0 & 17.6 & 0.1629 \\
\hline Benzaldehyde & 546.0 & 440.1 & 645.5 & 145.8 & 99.0 & 332.9 & 0.0485 \\
\hline Octanal & 163.9 & 91.8 & 221.7 & 15.4 & 12.7 & 20.5 & 0.0024 \\
\hline
\end{tabular}

and $1.87 \mathrm{nmol} \mathrm{L}^{-1}$, respectively, proving that among all the presenting methods, the one presented in this study was the most sensitive. Similarly, in our study LOD for octanal, benzaldehyde, 2-butanone and 4-heptanone were much lower than that presented by Calejo et al. ${ }^{16}$ Compared to LOD obtained by Mochalski et al., ${ }^{6}$ a slightly lower LOD was obtained in our study for 2-butanone, 4-heptanone and 2-heptanone. The lowest sensitivity in our study was obtained for $p$-cresol. However, this is still a satisfactory result compared to high-performance liquid chromatography methods, electrochemical microneedles and fluorescence spectroscopy, as summarized by Usha \& Gupta. ${ }^{33}$ The same authors reported a sensor with a layer of a nanocomposite of zinc oxide and molybdenum sulfide, which obtained LOD of $28 \mathrm{nmol} \mathrm{L}^{-1}$ for $p$-cresol. ${ }^{33}$

\section{Method application}

To prove the applicability of the method to the real matrix of human urine, the developed protocol was applied for the determination and quantification of target analytes in urine samples of healthy children and children with CD. The results revealed that the optimized method was applicable to the quantification of biomarkers in urine samples from both healthy children and children with $\mathrm{CD}$. To prove the concept that the urinary profile of VOCs can be used to discriminate between $\mathrm{CD}$ and healthy children, a partial least squaresdiscriminant analysis (PLS-DA) was applied. A score scatter plot was obtained for CD $(N=9)$ and healthy children $(N=9)$ (Fig. 3a). Even though a limited number of samples were analyzed, the PLS-DA score scatter plot showed a distinction between healthy children (red triangles) and children with $\mathrm{CD}$ (blue dots). Samples from CD children were present mainly in 1 quadrant, while samples from healthy children showed a spread in quadrants 2, 3 and 4 (Fig. 3a). The distribution of healthy children can be explained by a more diverse diet compared to a gluten-free diet followed by children with CD.
Variable Importance in the Projection (VIP) scores were measured to summarize the contribution each variable makes to the model (Fig. 3b). The higher value of the VIP score, the greater the contribution to the group separation. Based on VIP scores, 1,3-di-tert-butylbenzene, 2,3-butanedione, 2-heptanone, dimethyl disulfide and octanal were found to be discriminative compounds for CD vs. healthy children (VIP score above 1). Also, 4-heptanone, 2-butanone and hexanal have important contributions in the separation (VIP score above 0.83).

The concentration of the analyzed VOCs and the results from the non-parametric Mann-Whitney test are presented in Table 3 . Thiophene was detected in only one child with $\mathrm{CD}$, whilst the concentrations of 2-pentylfuran, dimethyl trisulfide and $p$ cresol were below the LOQ. 1,3-Di-tert-butylbenzene was only found in children with $\mathrm{CD}$, while trans-3-octen-2-one was observed in quantitative amounts only in healthy children. The concentrations of VOCs with high VIP scores were significantly different $(p<0.05)$ between healthy and CD children. The concentration of 2,3-butanedione, 2-butanone, dimethyl disulfide, hexanal, 4-heptanone, 2-heptanone and octanal was much higher in healthy children compared to $\mathrm{CD}$ children. It can be explained by the gut microbiota dysbiosis observed in $\mathrm{CD}$, as many VOCs are produced during colonic fermentation. ${ }^{15}$

Based on the performed analysis, it is suggested that 1,3-ditert-butylbenzene, 2,3-butanedione, 2-butanone, dimethyl disulfide, hexanal, 4-heptanone, 2-heptanone and octanal can be among the most significant biomarkers of $\mathrm{CD}$.

\section{Conclusions}

In the presented work, optimization of a protocol for the quantitative analysis of 15 selected urinary biomarkers of $\mathrm{CD}$, representing different chemical classes, was performed. This methodology proved to be simple, rapid and convenient to be included in the routine analysis of urine samples. The statistical 
experimental design was successfully applied to optimize the extraction parameters and study the most significant factors and interactions between them. When the method was applied to urine samples from healthy children and children with CD, it was demonstrated that the proposed method could distinguish the two groups of children based on the profile of urinary biomarkers. The potential of the method could be further explored by studying a larger group of participants to establish diagnostic protocols for CD. Moreover, the optimized method will be used to evaluate the effect of gluten-free diet modification on the profile of VOCs in urine of children with CD.

\section{Authors contributions}

ND conceived and designed the research. ND secured funding and administrated the project. HAA performed the statistical experimental design step. ND conducted all analytical experiments. UKK provided urine samples from children with CD. ND collected samples from healthy volunteers. ND and HAA analyzed data and interpreted results. ND wrote the draft of the manuscript. HAA contributed to the further writing of the manuscript. All authors read and approved the final version of the manuscript.

\section{Conflicts of interest}

There are no conflicts to declare.

\section{Acknowledgements}

The research was supported by the Polish National Science Centre through project PRELUDIUM 11 (project number: 2016/ 21/N/NZ9/01510).

\section{References}

1 M. M. Leonard, A. Sapone, C. Catassi and A. Fasano, J. Am. Med. Assoc., 2017, 318, 647-656.

2 F. Benkebil, C. Combescure, S. I. Anghel, C. Besson Duvanel and M. G. Schäppi, World J. Gastroenterol., 2013, 19, 51115117.

3 T. Majchrzak, W. Wojnowski, G. Piotrowicz, J. Gębicki and J. Namieśnik, TrAC, Trends Anal. Chem., 2018, 108, 38-49.

4 C. J. S. Probert, I. Ahmed, T. Khalid, E. Johnson, S. Smith and N. Ratcliffe, J. Gastrointestin. Liver Dis., 2009, 18, 337-343.

5 F. Buljubasic and G. Buchbauer, Flavour Fragrance J., 2015, 30, 5-25.

6 P. Mochalski and K. Unterkofler, Analyst, 2016, 141, 47964803.

7 C. L. Silva, M. Passos and J. S. Cmara, Br. J. Cancer, 2011, 105, 1894-1904.

8 R. Guadagni, N. Miraglia, A. Simonelli, A. Silvestre, M. Lamberti, D. Feola, A. Acampora and N. Sannolo, Anal. Chim. Acta, 2011, 701, 29-36.

9 A. P. Antón, A. M. C. Ferreira, C. G. Pinto, B. M. Cordero and J. L. P. Pavón, J. Chromatogr. A, 2014, 1367, 9-15.
10 R. P. Arasaradnam, N. Ouaret, M. G. Thomas, N. Quraishi, E. Heatherington, C. U. Nwokolo, K. D. Bardhan and J. A. Covington, Inflammatory Bowel Dis., 2013, 19, 999-1003. 11 R. B. M. Aggio, B. De Lacy Costello, P. White, T. Khalid, N. M. Ratcliffe, R. Persad and C. S. J. Probert, J. Breath Res., 2016, 10, 017106.

12 N. D. McGuire, R. J. Ewen, B. De Lacy Costello, C. E. Garner, C. S. J. Probert, K. Vaughan and N. M. Ratcliffe, Meas. Sci. Technol., 2014, 25, 065108.

13 R. Di Cagno, M. De Angelis, I. De Pasquale, M. Ndagijimana, P. Vernocchi, P. Ricciuti, F. Gagliardi, L. Laghi, C. Crecchio, M. Guerzoni, M. Gobbetti and R. Francavilla, BMC Microbiol., 2011, 11, 219.

14 R. Di Cagno, C. G. Rizzello, F. Gagliardi, P. Ricciuti, M. Ndagijimana, R. Francavilla, M. E. Guerzoni, C. Crecchio, M. Gobbetti and M. De Angelis, Appl. Environ. Microbiol., 2009, 75, 3963-3971.

15 R. P. Arasaradnam, E. Westenbrink, M. J. McFarlane, R. Harbord, S. Chambers, N. O'Connell, C. Bailey, C. U. Nwokolo, K. D. Bardhan, R. Savage and J. A. Covington, PLoS One, 2014, 9(10), e107312.

16 I. Calejo, N. Moreira, A. M. Araújo, M. Carvalho, M. De Lourdes Bastos and P. G. De Pinho, Talanta, 2016, 148, 486-493.

17 M. Kusano, E. Mendez and K. G. Furton, Anal. Bioanal. Chem., 2011, 400, 1817-1826.

18 C. M. Weber, M. Cauchi, M. Patel, C. Bessant, C. Turner, L. E. Britton and C. M. Willis, Analyst, 2011, 136, 359-364.

19 S. Bouatra, F. Aziat, R. Mandal, A. C. Guo, M. R. Wilson, C. Knox, T. C. Bjorndahl, R. Krishnamurthy, F. Saleem, P. Liu, Z. T. Dame, J. Poelzer, J. Huynh, F. S. Yallou, N. Psychogios, E. Dong, R. Bogumil, C. Roehring and D. S. Wishart, PLoS One, 2013, 8, e73076.

20 A. Pysanenko, T. Wang, P. Španěl and D. Smith, Rapid Commun. Mass Spectrom., 2009, 23, 1097-1104.

21 A. Naccarato, E. Gionfriddo, R. Elliani, G. Sindona and A. Tagarelli, J. Chromatogr. A, 2014, 1372, 253-259.

22 S. Risticevic, H. Lord, T. Górecki, C. L. Arthur and J. Pawliszyn, Nat. Protoc., 2010, 5, 122-139.

23 B. d'Acampora Zellner, C. Bicchi, P. Dugo, P. Rubiolo, G. Dugo and L. Mondello, Flavour Fragrance J., 2008, 23, 297-314.

24 G. A. Mills and V. Walker, J. Chromatogr. B: Biomed. Sci. Appl., 2001, 753, 259-268.

25 R. B. M. Aggio, A. Mayor, S. Coyle, S. Reade, T. Khalid, N. M. Ratcliffe and C. S. J. Probert, Chem. Cent. J., 2016, 10, 9.

26 R. Cozzolino, L. De Magistris, P. Saggese, M. Stocchero, A. Martignetti, M. Di Stasio, A. Malorni, R. Marotta, F. Boscaino and L. Malorni, Anal. Bioanal. Chem., 2014, 406, 4649-4662.

27 K. Schmidt and I. Podmore, J. Mol. Biomarkers Diagn., 2015, 6, 253.

28 S. Smith, H. Burden, R. Persad, K. Whittington, B. De Lacy Costello, N. M. Ratcliffe and C. S. Probert, J. Breath Res., 2008, 2, 037022. 
29 R. Shirley, SPME/GC Analyses of Sulfur Gases and VOCs, Using a New Carboxen/PDMS Fiber, 1999, vol. 16.

30 M. Monteiro, M. Carvalho, R. Henrique, C. Jerónimo, N. Moreira, M. De Lourdes Bastos and P. G. De Pinho, Eur. J. Cancer, 2014, 50, 1993-2002.
31 F. Chen, C. Wang, M. Zhang, X. Zhang, Y. Liu, J. Ye and Q. Chu, Talanta, 2014, 119, 83-89.

32 D. Song, Y. Gu, L. Liang, Z. Ai, L. Zhang and H. Xu, Anal. Methods, 2011, 3, 1418-1423.

33 S. P. Usha and B. D. Gupta, Biosens. Bioelectron., 2018, 101, 135-145. 